
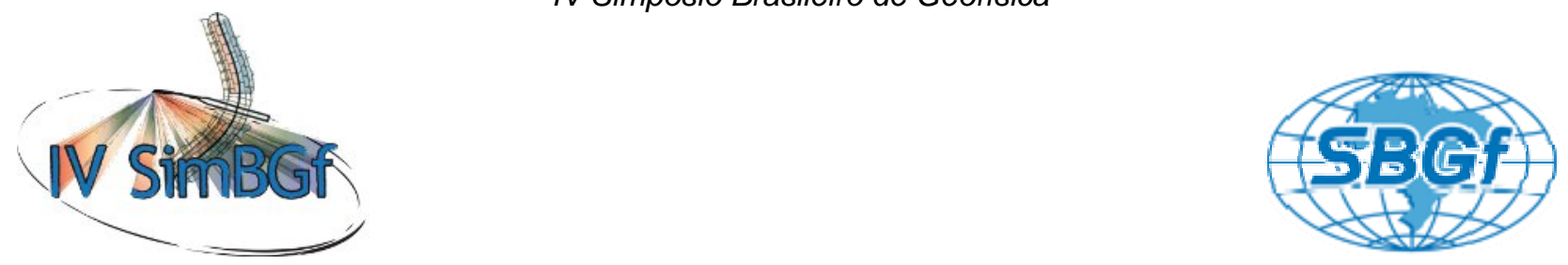

\title{
Estudo comparativo entre fontes sísmicas de alta resolução que utilizam tecnologia Chirp e sistemas paramétricos
}

Marina de Andrade Iguatemy; Arthur Ayres Neto

Universidade Federal Fluminense

Copyright 2008, SBGf - Sociedade Brasileira de Geofísica

Este texto foi preparado para a apresentação no IV Simpósio Brasileiro de Geofísica, Belém, 14 a 17 de novembro de 2010. Seu conteúdo foi revisado pelo Comitê Técnico do IV SimBGf, mas não necessariamente representa a opinião da SBGf ou de seus associados. É proibida a reprodução total ou parcial deste material para propósitos comerciais sem prévia autorização da SBGf.

\section{Resumo}

This paper aims to compare two types of high-resolution seismic sources: Chirp source, which emits a kind of controlled wave (FM pulse) that has the characteristic of knowing the pulses that were sent by first-order autocorrelation, and sources that use parametric systems, where two high frequencies emitted simultaneously interact to generate a new frequency equal to the difference of the two previous ones. The signal generated by the parametric system has high directivity and absence of side lobes. The results of two surveys conducted in Barra do Furado in the municipality of Quissamã northern coast of Rio de Janeiro, will be present also. The results presented shows considerable differences between sources concerning penetration and vertical resolution.

\section{Introdução}

A caracterização das sub-superfícies rasas das áreas subaquáticas (rios, lagos, áreas costeiras e plataforma continental) é extremamente importante, pois contribui tecnicamente para o planejamento das atividades, cada vez mais intensas e concentradas nestes complexos e sensíveis ambientes. Algumas destas atividades podem ser destacadas: o estabelecimento de obras de plataformas, a instalação de cabos e linhas de dutos, de prospecção de recursos minerais (petróleo, minerais, entre outros), a exploração de recursos pesqueiros, entre outras. Oliveira \& Brito (1998) discutem detalhadamente aspectos geológicos e geotécnicos relacionados com vários tipos de projetos desenvolvidos em ambientes subaquáticos. Métodos sísmicos de alta resolução têm sido utilizados intensamente no mapeamento do Quaternário, na investigação dos processos sedimentares, na engenharia costeira, na exploração mineral entre outros temas. Ao contrário da investigação sísmica convencional, a sísmica de reflexão de alta resolução lida com meios geológicos que comumente apresentam baixos contrastes de impedância acústica, baixa relação sinal ruído, além da propriedade do meio geológico se constituir em um forte atenuador de sinais de alta freqüência. A investigação destes meios exige utilização de fontes acústicas que emitem sinais com espectros de freqüência mais altos, o que torna a

rugosidade das superfícies, ou das interfaces, propriedades físicas relevantes. Os métodos sísmicos de alta resolução se destacam quando comparados com outros métodos geofísicos. Este destaque tem como base algumas características especiais, dentre as quais podem ser ressaltadas;

- a grande precisão e resolução dos sinais sísmicos; - o produto obtido em campo, em tempo real, é um perfil contínuo onde se identificam os refletores (contatos geológicos) em subsuperfície, à medida da evolução do levantamento;

- os perfis obtidos em campo podem ser interpretados praticamente como uma seção geológica sobrelevada, possibilitando que decisões possam ser tomadas durante a própria aquisição de dados;

- os levantamentos podem ser conduzidos em embarcações de pequeno e médio porte que se movem a velocidades superiores a $6 \mathrm{~km} / \mathrm{h}$, o que possibilita rápida cobertura da área investigada;

O foco principal é realizar uma análise de dois tipos diferentes de fontes sísmicas (baseadas em tecnologia CHIRP e em sistemas paramétricos) e avaliar qual dessas fontes apresenta melhor eficácia quando aplicadas em diferentes abordagens, analisando vantagens, desvantagens, das fontes sísmicas aplicadas a levantamento de áreas subaquáticas, e então orientar um melhor aproveitamento e a melhor qualidade do produto final da investigação, propondo possíveis critérios de escolha de fontes sísmicas para a solução mais apropriada das questões geológicas envolvidas na área de atuação.

\section{Metodologia/ Problema Investigado}

O Sistema Paramétrico é constituído de transdutores acústicos que utilizam um princípio onde a produção de sinais de baixa freqüência a partir da emissão de dois sinais simultâneos de freqüências ligeiramente diferentes e maiores (Grant \& Schreiber, 1990; Wunderlich, 2003). Devido a não-linearidades na propagação do som em altas pressões a interferência entre os dois sinais emitidos gera uma freqüência secundária. A freqüência secundária é gerada no centro do feixe incidente, que possui um estreito ângulo quando comparado com o feixe das freqüências primárias, devido à ausência de ecos laterais. O sistema Chirp corresponde a um tipo de fonte de alta resolução que utiliza tecnologia chirp, apresentando freqüência modulada (FM). Ele emite um pulso que varre uma janela de freqüências e uma de suas características é sua função de autocorrelação. Uma vantagem adicional dessa autocorrelação é que ele consegue um ganho de processamento de sinal sobre o ruído de fundo. A pesquisa de LeBlanc et al (1992) constatou que, de modo a igualar o desempenho de 
pulsos do sinal chirp, pulsos convencionais teriam que usar um pulso com uma potência de pico de 100 vezes maior.

Os dois tipos de fontes foram utilizados em um levantamento na área de Barra do Furado (Fig 1), município de Quissamã, Rio de Janeiro, onde a cobertura sedimentar é predominantemente siliciclástica, com presença de biodetritos e concreções espalhadas de forma aleatória sobre as diversas unidades de sedimentação.

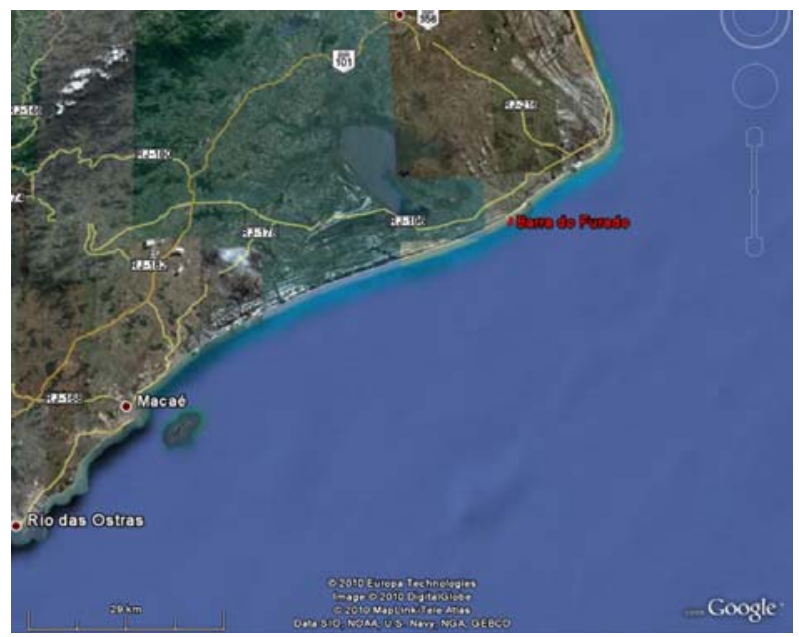

Figura 1: Área onde foi realizado o levantamento utilizando os sistemas chirp e paramétricos (Fonte: Google Earth).

Para entender o comportamento das respostas obtidas pelas duas fontes foram analisados alguns parâmetros importantes, tais como: Diretividade do feixe, que depende das dimensões do transdutor relacionado à freqüência do som emitido e resolução que entende-se a capacidade de um método geofísico detectar dois objetos distintos na superfície (resolução horizontal) ou em subsuperfície (resolução vertical) e está diretamente relacionada com o espectro de freqüências emitido pela fonte acústica.

\section{Resultados}

Foram analisados dois perfis de levantamento onde ocorrem à interseção dos dois equipamentos, Chirp e Paramétrico, com o objetivo de observar suas atuações em um meio predominantemente arenoso. Na figura 2, estão identificados os perfis, onde as linhas em vermelho correspondem ao levantamento chirp e a linha em verde corresponde ao levantamento utilizando o sistema paramétrico.

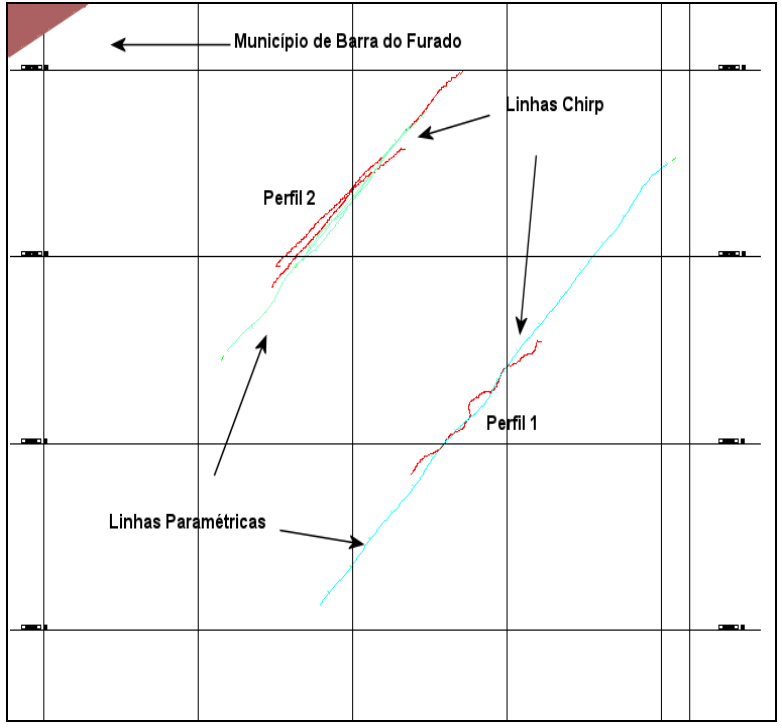

Figura 2: Área onde foram realizados os levantamentos utilizando os dois tipos de fontes, utilizando o sistema paramétrico e o chirp.

Foram utilizados diferentes padrões de visualização dos perfis, devido a possíveis variações de ganho durante a aquisição dos levantamentos. As tonalidades foram ajustadas para a melhor apreciação dos refletores. O software utilizado foi o Kingdom 8.4.

O perfil 1 (Fig.3) localiza-se na porção mais externa da área de levantamento. Os dados obtidos no perfil $1 \mathrm{com}$ os dois equipamentos apresentaram diferenças consideráveis. Em ambos os levantamentos, chirp e paramétrico, foi observado um fundo reflexivo, podendo estar associado a um fundo arenoso, o que resulta em uma baixa penetração do sinal acústico. Nos dados adquiridos com o equipamento paramétrico pode se observar alternância de refletores sub-superficiais ao longo da linha. Em alguns momentos foi observado uma intercalação de um segundo refletor em sub-superfície. Esse fato pode estar relacionado a mudanças de fácies sedimentares no fundo marinho ou nas camadas subsuperficiais, que acarretam na penetração ou não da onda acústica. Nos dados paramétricos também se observa a presença de pequenas hipérboles que no sistema chirp, não foram visualizadas. A presença dessas hipérboles pode estar relacionada ao estreito feixe emitido pelo sistema paramétrico, o que permite uma melhor visualização de estruturas de pequena escala sobre o fundo marinho (resolução horizontal). Em relação à resolução vertical, o método paramétrico também se mostrou mais eficiente, pois foi possível um melhor detalhamento dos primeiros refletores em subsuperfície, enquanto que nos dados chirp essa visualização se mostrou confusa de difícil mapeamento. Entretanto os dados chirp mostraram melhores resultados em relação a penetração. De acordo com a figura 3, nesta linha, foi possível a visualização de um refletor a cerca de $15 \mathrm{~ms}$ de profundidade e nos dados paramétricos o máximo foi de $4 \mathrm{~ms}$. Esse resultado também foi observado por Weber e Kuhn (1993), onde foi 
identificada uma melhor resolução vertical e horizontal do sinal acústico do sistema paramétrico, comparado ao sistema chirp, em regiões planas.

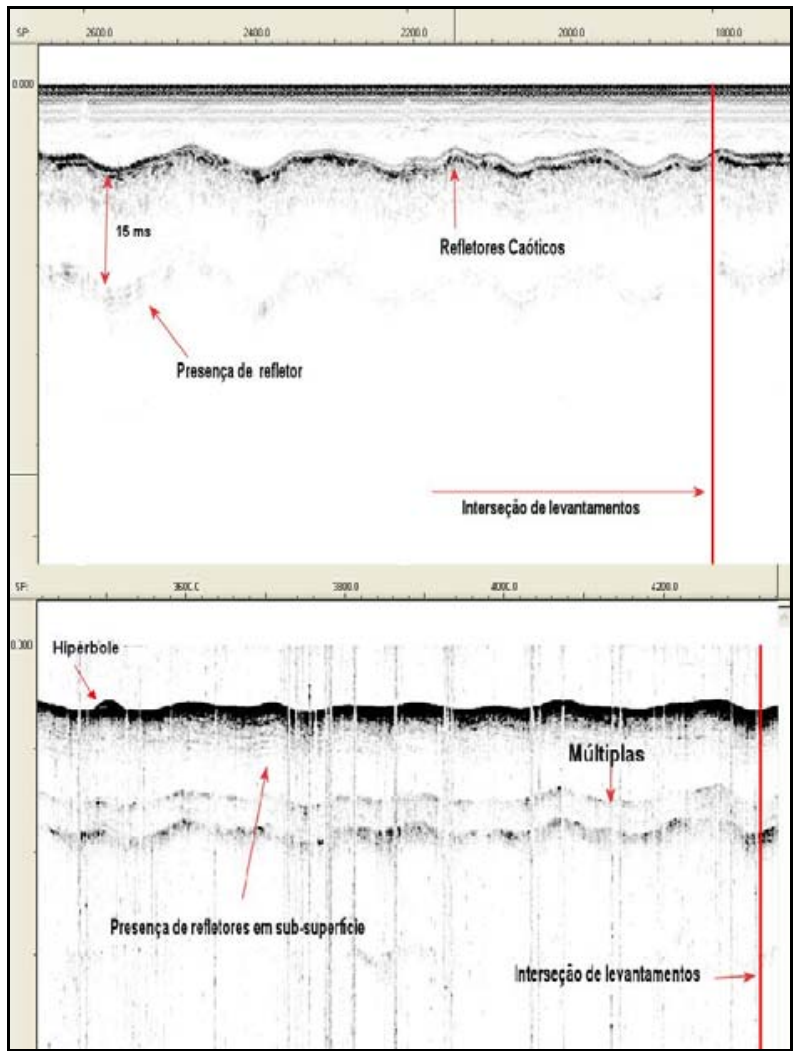

Figura 3 -Comparação de levantamentos, na região superior da figura, perfil 1, Chirp e abaixo, perfil Paramétrico.

O Perfil-2 (Fig. 4) localiza-se na porção mais próxima à costa da área de levantamento, O sistema paramétrico novamente se mostrou mais eficiente em relação à resolução vertical, onde foi possível visualizar com maior nitidez um refletor em sub-superfície bem próximo ao fundo marinho.Também foi possível observar pequenas hipérboles que podem estar relacionadas a pequenas estruturas sedimentares (ripples) ou enterradas sobre 0 fundo marinho. Devido ao pequeno ângulo do sinal acústico irradiado pelo sistema paramétrico, pode se observar um aumento considerável da resolução espacial reduzindo assim a dispersão do sinal já na primeira camada de interação, favorecendo assim a visualização da morfologia sedimentar do local de estudo. No sistema chirp pode-se observar novamente a baixa resolução horizontal e vertical do sinal acústico, apresentando pouca visibilidade para o mapeamento do segundo refletor em sub-superfície. Em relação à penetração, foram visualizados refletores com cerca de $12 \mathrm{~ms}$ no sistema chirp e no sistema paramétrico não ultrapassam de 4 ms ( fig. 4 ).

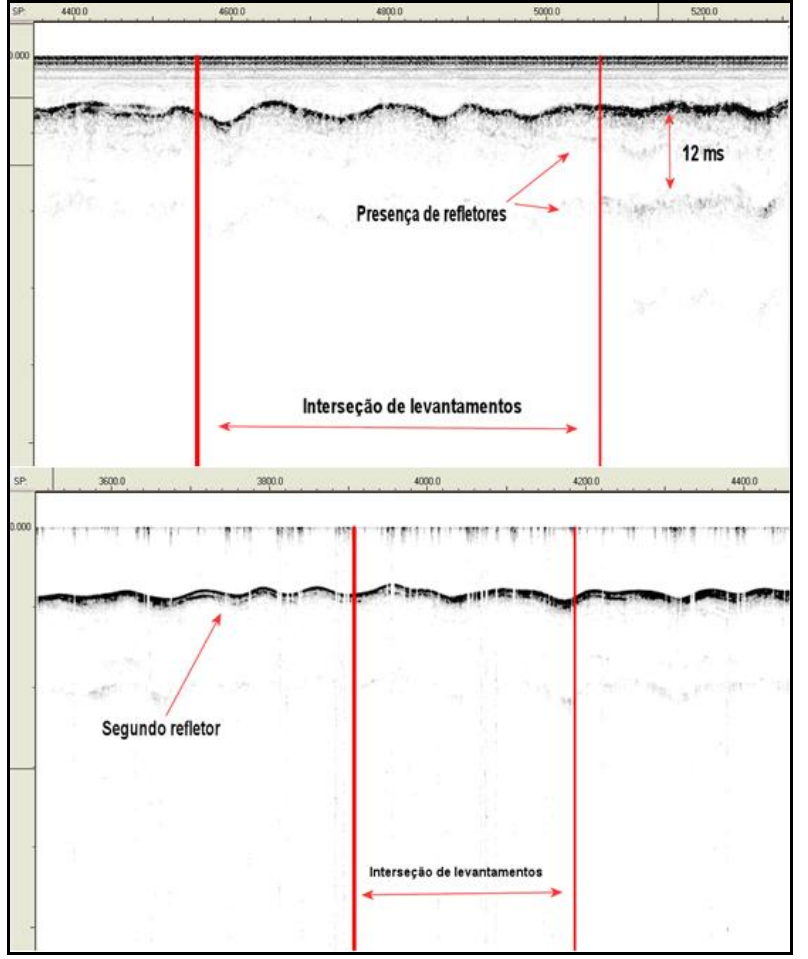

Figura 4 -Comparação de levantamentos, na região superior da figura, perfil 2 Chirp e abaixo, perfil Paramétrico.

\section{Discussão e Conclusões}

Após a comparação qualitativa dos perfis sísmicos adquiridos, pode-se constatar que o equipamento paramétrico permite um melhor reconhecimento de refletores sísmicos próximos ao fundo marinho (melhor resolução vertical) quando comparado ao sistema Chirp. Nota-se que o estreito feixe emitido pela associação de duas altas freqüências, gerando uma freqüência secundária, mostra-se uma ferramenta poderosa nas identificações de camadas sedimentares muito rasas. Nos dados do sistema paramétrico pode ser observados melhores condições de identificação de hipérboles, que podem representar pequenas estruturas no fundo marinho, em superfície e em sub-superficie. Em termos de engenharia, no caso de investigação de dutos enterrados, isso faz com que o sistema paramétrico tenha uma importância considerável, pois quanto melhor a identificação do topo da hipérbole, melhor se extrai a cota de enterramento dos dutos. Esses resultados estão de acordo, em relação a resolução sísmica, com o trabalho de Weber E. M. \& Kuhn G.(1993), que também realizaram comparações entre os dois tipos de fontes em lugares equivalentes. Entretanto em relação à penetração, os autores supra citados também obtiveram melhores resultados no sistema paramétrico, porém o fundo marinho levantado pelos mesmos se tratava de lamas intercaladas, isso de alguma forma pode ter causado a divergência de resultados. Os dados obtidos com o sistema Chirp apresentaram melhores resultados 
quando comparados com o sistema paramétrico, atingindo refletores de aproximadamente $15 \mathrm{~ms}$. Para entender melhor o comportamento de ambas as fontes e conseguir diagnosticar quais das mesmas se mostra mais eficiente seria necessário o mapeamento em áreas mais profundas e que apresentassem variações de relevo para assim poder analisar parâmetros relevantes para a se tomar a decisão de qual fonte se faz mais proveitosa. Além disso, devem ser adotados critérios mais rígidos no levantamento, mantendo maior controle dos parâmetros de aquisição, a fim de que possa ser realizada uma comparação mais quantitativa entre os dois sistemas.

\section{Referências}

GRANT, J.A. \& R. SCHREIBER. 1990. Modern swathe sounding and sub-bottom profiling Technology for research applications: The Atlas Hydrosweep and Parasound Systems. Mar. Geophys. Res., 12(1-2):9-19

LeBlanc, L.R., Mayer, L., Rufino, M., Schock, .G. \& King,J., 1992. Marine sediment classification using chirp sonar. J.Accoust. Soc, Am, 91, 107-115

OLIVEIRA, A.M.S. \& Brito S.N.A.. 1998. Geologia de Engenharia. São Paulo, ABGE. 586p.

WEBER E. M. \& KUHN G. 1993. Acoustical characterization of sediments by Parasound and $3.5 \mathrm{kHz}$ systems: Related sedimentary processes on the southeastern Weddell Sea continental slope, Antarctica , Marine Geology, 113

WUNDERLICH, J. \& G. WENDT. 2004. High-Resolution Echo-Sounding and Estimation of Sediment Properties using Nonlinear Acoustics. In: Near Surface 2004 European Meeting of environmental and Engineering Geophysics, 10. 